\title{
Quantitative STEM and EELS for the Study of Alloy Nanoparticles, Support Materials, and Graphene for Fuel Cell Applications
}

\author{
M.C.Y. Chan, F. Nan, S. Prabhudev, S. Stambula, M. Bugnet, N. Gauquelin and G.A. Botton \\ Department of Materials Science and Engineering, McMaster University, Hamilton, Ontario, \\ Canada, L8S 4M1.
}

The study of various catalyst materials has significantly been improved with the developments of electron microscopes equipped with aberration correctors. Recent reviews have demonstrated the benefits of improved resolution for chemical analysis and imaging [1]. These benefits stem from the improved stability of the instrumentation thus making quantitative analysis of strain and signal intensities possible. With improved electron energy loss spectroscopy capabilities, (using monochromators, high brightness sources) it is possible to simultaneously obtain chemical analysis at the atomic level and structural analysis using high-angle annular dark-field (HAADF) imaging. Here, an overview of recent examples related to the study of various catalyst nanoparticles used for fuel cells, their hybrid supports, and novel substrates such as graphene will be presented.

Experiments were carried out on two aberration-corrected microscopes (both FEI Titan 80-300) [2,3] equipped with electron monochromators providing $0.1 \mathrm{eV}$ (or better) energy resolution and a Tecnai Osiris comprising a high efficiency energy dispersive X-ray detector. With these instruments we have demonstrated atomic-resolved mapping [2], and mapping the lowest energy loss features currently reported in the literature [3]. We studied a variety of samples including Pt/transition metal catalysts and their support materials and graphene based catalysts.

Comparison between HAADF theory and experiments on PtFe nanoparticles [4] has shown that it is possible to detect the ordering in the core of nanoparticles and, very significantly, that the ordering is not maintained on the surface of the same nanoparticles (figure 1). Image simulations also demonstrate that the shell of these nanoparticles is of approximately 2-3 atomic layers and $\mathrm{Pt}$ enriched. Detailed analysis of the atomic displacements in proximity of the surface has also shown that the atoms are very significantly displaced from their expected position extrapolated from atoms just a few layers away from the particle surfaces (figure 2). Detailed chemical analysis of particles subjected to electrochemical cycling, using energy-dispersive X-ray spectrometry with large solidangle Si drift detectors, shows a significant change in the size distribution and that the surface of the particles becomes more significantly enriched in Pt (figure 3) [5]. Compositional measurements carried out as a function of particle sizes also show that the smaller particles become more drastically Fe deficient [5].

Electron tomography also plays a major role in the analysis of complex catalysts. With this technique, it is possible to detect the oxide in hybrid carbon/oxide supports along with the 3D relative distribution (figure 4). With high-resolution electron energy loss spectroscopy, evidence of interactions between the catalysts and the support materials have also been studied. During the presentation, various recent examples of these techniques and materials will be demonstrated [6].

\section{References:}

[1] J. Liu, Chemcatchem, 3, pp 934-948, (2011). 
[2] G.A. Botton, S. Lazar and C. Dwyer, Ultramicroscopy, 110, 926- 934, (2010).

[3] D. Rossouw and G.A. Botton, Physical Review Letters 110, 066801 (2013).

[4] M.C.Y. Chan, et al, Nanoscale 4 (22), 7273-7279 (2012)

[5] C. Bock, et al. Chemcatchem In press. DOI: 10.1002/cctc.201200614

[6] This work is supported by the NSERC (Canada) and was carried at the Canadian Centre for Electron Microscopy, a facility supported by NSERC and McMaster University. We are grateful to C. Bock for providing samples related to this work.

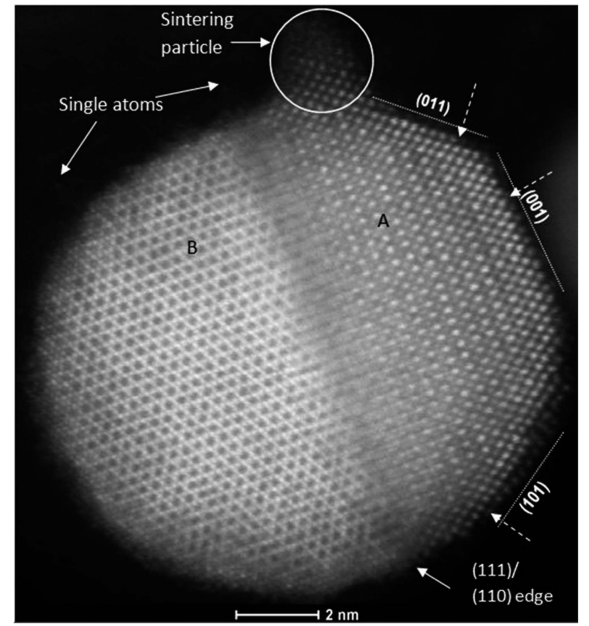

Figure 1. HAADF STEM image of a PtFe nanoparticle formed from the coalescence of smaller particles. Region A shows the [110] projection and region $\mathrm{B}$ the [111] projection.

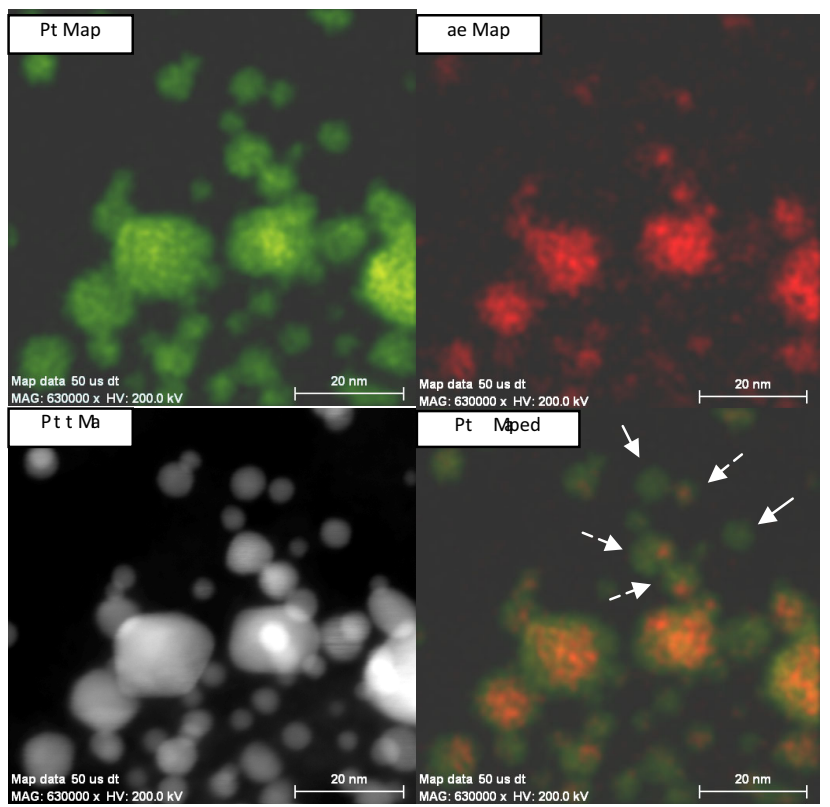

Figure 3. Energy dispersive X-ray spectroscopy map of PtFe nanoparticles following electrochemical cycling.
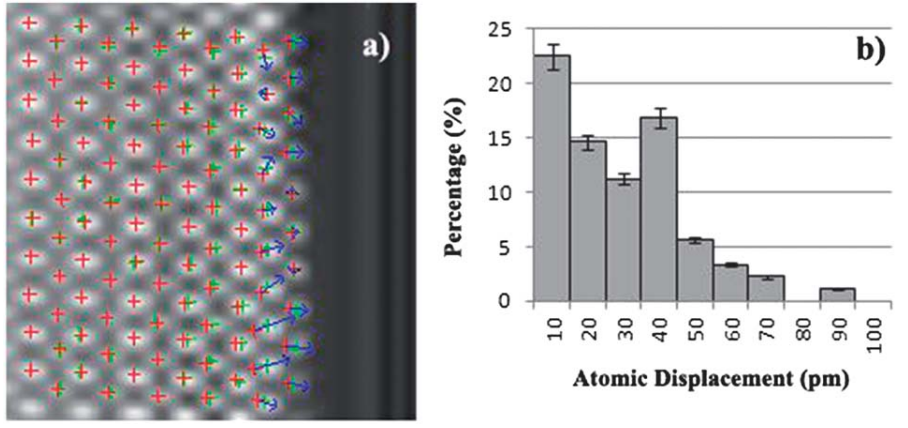

Figure 2. Quantitative measurement of atomic spacing in proximity of the nanoparticle surface (a) and histogram of atomic displacements (b).

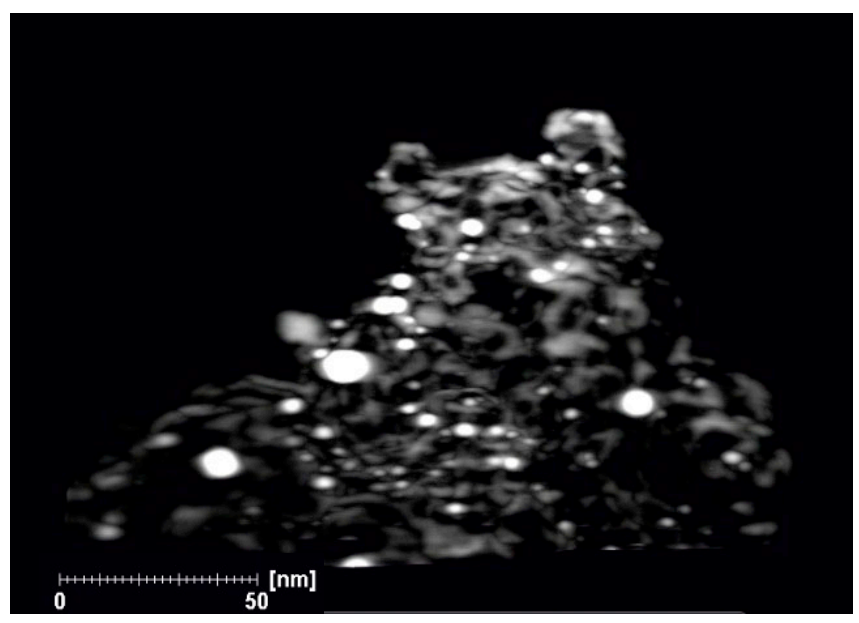

Figure 4. Tomographic reconstruction of complex oxide support with Pt catalysts 